\title{
Effective maNagement of Depression among Patients witH cANCEr (ENHANCE): A Protocol for a Hybrid Systematic Review and Network Meta-Analysis of Randomized Controlled Trials of Interventions for Depressive Symptoms.
}

Maria Margareta Pertl ( $\square$ mariapertl@rcsi.ie)

Royal College of Surgeons in Ireland https://orcid.org/0000-0001-7326-2763

\section{Sergio Perez,}

Trinity College Dublin: The University of Dublin Trinity College

\section{Sonya Collier}

St James Hospital

\section{Emer Guinan}

Trinity College Dublin: The University of Dublin Trinity College

\section{Garret Monahan}

Royal College of Surgeons in Ireland

\section{Katie Verling}

Royal College of Surgeons in Ireland

\section{Emma Wallace}

Royal College of Surgeons in Ireland

\section{Aisling Walsh}

Royal College of Surgeons in Ireland

\section{Frank Doyle}

Royal College of Surgeons in Ireland

\section{Protocol}

Keywords: Systematic review, network meta-analysis, depression; cancer, pharmacotherapy, psychotherapy, exercise, collaborative care, complementary and alternative medicine, public and patient involvement.

Posted Date: December 20th, 2021

DOI: https://doi.org/10.21203/rs.3.rs-1128439/v1

License: (c) (i) This work is licensed under a Creative Commons Attribution 4.0 International License. Read Full License 


\section{Abstract}

\section{Background:}

Depression is common among patients with cancer and is associated with lower treatment participation, lower satisfaction with care, poorer quality of life, greater symptom burden, and higher healthcare costs. Various types of interventions (e.g., pharmacological, psychotherapy) are used for the treatment of depression. However, evidence for these among patients with cancer is limited. Furthermore, the relative effectiveness and acceptability of different approaches is unknown because a direct comparison between all available treatments has not been carried out. We will address this by conducting a network meta-analysis (NMA) of interventions for depression among people with cancer using a hybrid overview of reviews and systematic review methodology.

\section{Methods:}

We will search for and extract data from systematic reviews of randomised controlled trials (RCTs) of depression interventions for patients with cancer from inception, before performing a supplemental search for more recent RCTs. We will include RCTs comparing pharmacological, psychotherapy, exercise, combination therapy, collaborative care, or complementary and alternative medicine interventions with pill placebo, no treatment, waitlist, treatment as usual, or minimal treatment control groups, or directly in head-to-head trials, among adults who have a current or previous diagnosis of cancer and elevated depressive symptoms (scores above a cut-off on validated scales or meeting diagnostic criteria). Our primary outcomes will be change in depressive symptoms (standardised mean difference) and intervention acceptability (\% who withdrew). Our secondary outcomes will be 6-month change in depressive symptoms, health-related quality of life, adverse events and mortality. We will independently screen for eligibility, extract data, and assess risk of bias using the RoB2 tool. We will use frequentist random-effects multivariate NMA in Stata, Rankograms and surface under the cumulative ranking curves to synthesize evidence and obtain a ranking of intervention groups. We will explore heterogeneity and inconsistency using local and global measures and evaluate the credibility of results using the Confidence in NEtwork Meta-Analysis (CINeMA) framework.

\section{Discussion:}

Our findings will provide the best available evidence for managing depression among patients with cancer. Such information will help to inform clinical guidelines, evidence-based treatment decisions and future research by identifying gaps in the current literature.

\section{Systematic review registration:}

Submitted to PROSPERO (record number: 290145), awaiting registration

\section{Background}

Patients with cancer have a five-fold increased odds of depressive symptoms compared to the general population, with one in four experiencing clinically significant symptoms (1). Moreover, the risk of major depression in patients with cancer is two-to-three times the estimated prevalence in the general population (2). Prevalence rates span from $16-25 \%$ (3), with estimates varying widely due to different methodological standards, the various screening methods and diagnostic instruments used, as well as cancer type and severity (3-5). Determining the presence of depression among patients with cancer is also particularly challenging because it can be difficult to distinguish between normal psychological distress and sadness in response to cancer and clinical depression (6). In addition, symptoms 
associated with cancer and side-effects associated with its treatment (e.g., fatigue, weight loss and sleep disturbance) overlap with the diagnostic criteria for depression (6). Nevertheless, identifying and treating depression is of critical importance, not only for the welfare and quality of life of people with cancer but also for maximising the effectiveness, efficiency and acceptability of cancer treatments.

Depression among patients with cancer has been associated with lower treatment participation; lower satisfaction with care and quality of life; higher levels of anxiety, pain, fatigue; and higher healthcare costs, resource use and mortality (7-9). Furthermore, even depressive symptoms in the absence of a formal diagnosis of major depression have been found to be an independent risk factor for mortality and disease-related burden (e.g., 9, 10, 11, 12). Therefore, identifying treatments for depression that are effective and acceptable to patients with cancer is of key clinical importance, and greater awareness of the best management strategies may also encourage health professionals to more routinely assess and treat depression in clinical practice (13).

Many different types of interventions are used, often in combination, for the treatment of depression among patients with cancer (14). These include psychotherapies, such as Cognitive Behavioural Therapy (CBT), problem solving therapy, supportive therapy, counselling, and group therapy; pharmacological interventions, including antidepressants and stimulants; exercise interventions; and collaborative care interventions, which involve active collaboration between various health professionals (e.g., medical doctors, case managers, and mental health specialists) and the patient in managing the problem; as well as complementary and alternative medicine (CAM) interventions, such as acupuncture (14-19). However, evidence for the effectiveness, cost-effectiveness and acceptability of these interventions among patients with cancer is limited and mixed $(16,18,20)$. As a result, implications for practice are unclear and clinical guidelines have had to draw on data from depression interventions for the general population rather than cancer specific studies $(18,20)$. However, the benefits of and adverse effects associated with interventions may be different among patients with cancer and the general population (16). Furthermore, since there is no evidence for the superiority of one intervention over another, guidelines have been limited to a large extent to broad statements on the general effectiveness of various approaches (19).

Obtaining information on the relative effectiveness and acceptability of different types of interventions for depression would enhance the provision of psycho-oncological care by providing guidance to clinicians around what type of interventions are most appropriate and providing policy-makers with excellent evidence for resource allocation. Such data are not available as direct comparisons between all available depression treatments have not been carried out; nevertheless, given the plethora of randomised controlled trials (RCTs) and meta-analyses that have evaluated specific interventions for depression among patients with cancer (e.g., 16, 21, 22), there are sufficient data for combined direct and indirect comparisons of existing interventions using Network meta-analysis (NMA).

NMA is an advanced technique that can fill gaps in the literature by making indirect comparisons between treatment options that have not consistently been made in head-to-head formats and can generate hierarchies of which treatments are the best, second best, and so on in terms of outcomes of interest $(23,24)$. For example, it has previously been used to compare the efficacy and acceptability of antidepressant drugs for the treatment of major depressive disorder (25). Furthermore, it can compare interventions that are very different in nature, such as medication and exercise (e.g., 26, 27), even when comparator groups may differ (Doyle et al., 2019, 2021). Such crucial information is urgently needed by clinicians and policy-makers but cannot be provided using conventional meta-analysis. Indeed, there is growing recognition that systematic reviews based on NMA constitute the highest level of evidence in treatment guidelines (Leucht et al., 2016). We propose to conduct an NMA summarizing the current literature on the treatment of depression among patients with cancer and ranking competing interventions in terms of 
efficacy and acceptability. A comparison of the efficacy of interventions for depression among patients with coronary artery disease has already been completed and has informed our methodological approach (27).

\section{Objectives}

The main objective of this NMA is to compare the efficacy and acceptability of established treatments for managing depression among patients who have had a diagnosis of cancer. The primary research questions are (1) What treatments are the most effective in reducing depressive symptoms among patients with cancer and (2) What treatments are the most acceptable for managing depressive symptoms among patients with cancer? Using the Patient, Intervention, Comparison, Outcome (PICO) procedure, our criteria to search for studies to answer these questions will be:

- Participants: (a) adult participants aged 18 years and over; (b) with a current or previous diagnosis of any cancer; (c) elevated depressive symptoms (i.e., scoring above the cut-off criteria on a validated depression screening instrument or meeting the diagnostic criteria on a standardised clinical diagnostic interview [e.g., the DSM-5 (28)] for assessing major depressive disorder, adjustment disorder, or dysthymic disorder) at the time of study entry; (d) enrolled in an RCT targeting depressive symptoms in any setting.

- Interventions: any established treatment for depression including pharmacotherapy; psychotherapy; exercise; combination therapy; and collaborative care; as well as complementary and alternative medicine (CAM) interventions.

- Comparison: any appropriate comparator group including usual care, placebo groups, no intervention, waitlist, attention control group, or another depression treatment.

- Outcomes:

\section{o Primary:}

(a) Efficacy-response (depressive symptom mean change scores between groups) after 8 weeks (range 4 - 12 weeks) from baseline on validated measures of depression;

(b) Acceptability (percentage of patients who discontinued with the intervention/comparator for any reason) at any stage.

\section{o Secondary:}

(a) Longer-term follow-up depression assessed at 26 weeks (range 20 and 30 weeks) on validated measures of depression;

(b) Health-related quality of life (HRQoL) or, if this is unavailable, general QoL (mean change scores between groups) after 8 weeks (range 4 - 12 weeks) from baseline on validated scales. If sufficient data are available, we will subdivide QoL into physical, social and emotional domains.

(c) Adverse events (the percentage of participants who leave the study as a result of intervention-related adverse events) occurring within 12 weeks of study commencement.

(d) Mortality (all-cause; the percentage of participants who die after or during the treatment [cancer related or otherwise]) for the longest duration of follow-up.

\section{Methods}


This protocol closely follows the methods of Doyle et al. (29) and the PRISMA extension statement for the reporting of systematic reviews incorporating NMA (30). We have provided a completed PRISMA-P checklist in Additional file 1. Public and Patient Involvement (PPI) helped us to refine the focus of the research questions and PPI will continue to play a key role in the running of this study. We have reported PPI in the development of this protocol using the short form of the Guidance for Reporting Involvement of Patients and the Public 2 (GRIPP2-SF; 31), which is available in Additional file 2. If any amendments to this protocol are necessary during the review process, we will add details and justifications for these to the registration record and report these in the final systematic review results report.

\section{Eligibility criteria}

\section{Study types}

Eligible studies will be RCTs of depression interventions administered to patients who are currently or have been previously diagnosed with cancer. The interventions of interest, based on clinical guidelines for the management of depression among patients with cancer and/or chronic physical health problems $(19,20,32,33)$ are pharmacotherapy, psychotherapy, exercise, combination therapies, and collaborative care interventions, as well as CAM approaches. As an outcome measure, studies using any of these interventions should employ a validated depression scale or diagnostic interview able to report a (potential) change in depression or depressive symptoms from baseline or pre- to post-treatment. Psychological interventions that are not established psychotherapies and are not delivered by professionally trained therapists will be excluded from the study. As recommended by Chaimani et al. (34), additional unspecified interventions that surface during the search process may be considered for inclusion in the network if the study meets the eligibility criteria and the inclusion of the intervention could serve to supplement the analysis by, for example, increasing the precision of the results. Studies included will be published (in English) in peer-reviewed journals, review articles or RCT registries.

\section{Participants}

Participants will be 18 years of age and over, and have a current or previous diagnosis of any cancer, and be at any stage of treatment (pre-treatment, active treatment, or post-treatment). Participants must be enrolled in an RCT targeting elevated depression as either a primary or secondary outcome, assessed using validated measures of depression symptoms at baseline and post intervention. We will exclude participants if they have (a) a diagnosis other than cancer, (b) antenatal/postnatal depression, (c) bipolar disorder or psychotic depression, or concurrent secondary psychiatric diagnoses. If some, but not all, of a study's participants are eligible for inclusion (e.g., if they include patients with cancer and patients with other diseases), then we will request the data for eligible patients only from the authors or, if $>80 \%$ of participants are eligible for the review, we will include the overall trial estimates.

\section{Intervention types}

We will include the following types of interventions; however, as recommended, unspecified interventions may also be included post hoc to improve the precision of the model (34):

\section{Pharmacotherapy}

Interventions in this category will comprise of any medicines used to treat the symptoms of depression. In assessing eligibility we will draw on clinical guidelines (e.g., 19, 20,32), being mindful of changes in recommended treatments over time. Examples include selective serotonin re-uptake inhibitors (SSRIs), serotonin-norepinephrine reuptake 
inhibitors (SNRIs), tricyclic anti-depressants, monoamine oxidase inhibitors, mirtazapine, agomelatine, etc. We will only include studies that randomised participants to pharmacotherapies within their licensed dose range (35).

\section{Psychotherapy}

We will adopt the inclusion criteria used by Cuijpers et al. (36) for the development of their complete database of trials on psychological treatments for depression (www.evidencebasedpsychotherapies.org). These are based on the definition of psychotherapy by Norcross (37): "Psychotherapy is the informed and intentional application of clinical methods and interpersonal stances derived from established psychological principles for the purpose of assisting people to modify their behaviours, cognitions, emotions, and/or other personal characteristics in directions that the participants deem desirable". Eligible interventions can be delivered by any therapist (including psychologists, nurses, social workers etc.) so long as they are trained to deliver the therapy and in any treatment format so long as they are facilitated (i.e., individual, group, telephone, guided self-help or couple therapy). As outlined in Cuijpers et al. (36), these fall into the following eight psychotherapy categories: (1) cognitive behaviour therapy (CBT), (2) behavioural activation therapy, (3) problem-solving therapy, (4) interpersonal psychotherapy, (5) psychodynamic therapy, (6) nondirective therapy, (7) third-wave therapies (e.g., acceptance and commitment therapy, mindfulness-based stress reduction, mindfulness-based cognitive therapy) and (8) life review therapy (for definitions and examples, see https://evidencebasedpsychotherapies.shinyapps.io/metapsy/_w_ed60cf71/variable_description.pdf).

\section{Combination therapy}

Interventions in this category will involve both psychotherapy and pharmacotherapy components.

\section{Exercise interventions}

We will include interventions that involve aerobic and/or resistance training exercise. Other types of exercise, such as yoga or Tai Chi, which are not delivered based on the prescription principles for exercise training, will not be included in this intervention group $(33,38,39)$.

\section{Collaborative care interventions}

Interventions in this category will involve a multi-component approach with active collaboration and enhanced interprofessional communication between different specialists and primary care providers (40).

\section{Complementary and alternative medicine (CAM)}

CAM interventions involve therapeutic approaches that are not usually included in conventional Western medicine (41), and they are used by patients in combination with or as alternative treatments for depression (42). In line with the National Centre for Complementary and Integrative Health and van der Watt et al. (43), we will include approaches covered in the following classifications: herbal interventions, nutritional supplements (e.g., vitamins or probiotics) and aromatherapy; cognitive interventions (e.g., hypnotherapy, imagery, and meditation); and physical interventions (e.g., tai chi, acupuncture and light therapy).

\section{Comparison groups}

To qualify for inclusion, RCTs must compare interventions with another appropriate comparator group such as treatment as usual (TAU), enhanced usual care, pill placebo control groups, no treatment, waitlist, attention control groups, or another depression treatment. Comparator groups will be considered separately as previous work has shown that these are not equivalent $(27,44,45)$. The nature of the control groups used can, for example, have a major influence on the results by impacting on risk of bias (such as attrition rates and blinding), heterogeneity, and 
effect sizes observed $(44,45)$. With this in mind, we will categorise comparators into the following three groups in line with recommendations $(46)$ and previous research $(27,29)$ :

1) Pill placebo (for drug trials);

2) No treatment, waitlist or treatment as usual;

3) Treatment control (defined as minimal treatment control, active comparator, and specific and non-specific factors treatment control).

We will contact authors for further information in instances where comparator groups are unclear and, if necessary, include an 'unclear' comparator group or exclude a study from the NMA if details on comparator groups are not available.

Figure 1 below shows a sample network plot, based on all of the possible depression interventions and comparison groups we plan to include.

\section{Outcomes}

\section{Primary outcomes}

We will include two primary outcomes, following the example of Doyle et al. (29) and Cipriani et al. (25):

1. Efficacy: depression (means and standard deviations [SDs]) measured using validated tools (diagnostic interviews or screening instruments), and summarized with standardized mean difference (SMD) from baseline to postintervention. The follow-up measure closest to 8 weeks will be used; however, measures within a range of $4-12$ weeks will be accepted. If multiple depression measures were included in a given study, scores on the Hamilton Depression rating scale (HAM-D) will be the used; if the HAM-D is not included then preference will be given to longer scales with better content validity $(35,47)$.

2. Acceptability: the percentage of participants who discontinue the intervention/comparator for any reason, at any stage.

\section{Secondary outcomes}

The secondary outcomes of interest are:

1. Longer-term follow-up efficacy: depression (means and SDs) measured using validated tools (diagnostic interviews or screening instruments), and summarized with SMD from baseline to follow-up (the measure closest to 26 weeks available will be used, between 20 and 30 weeks)

2. HRQoL: HRQoL scores (means and SDs) on physical, social and emotional domains summarized using SMD from baseline to post-intervention. As for the primary depression outcome, the measure closest to 8 weeks will be used with an acceptable range from 4 - 12 weeks. Generic QoL scores will be used when HRQoL scores are not available. 3. Adverse events: the percentage of participants who leave the study as a result of intervention-related adverse effects within 12 weeks of study commencement.

4. Mortality (all-cause): the percentage of participants who die after or during the treatment (cancer related or otherwise) for the longest duration of follow-up. 


\section{Search strategy and study selection}

As numerous systematic reviews exist on this topic (e.g., 16, 21, 22, 48), we will carry out a hybrid review of reviews and systematic review methodology (27). This approach involves first searching for relevant systematic reviews and extracting the pertinent references from these, before performing a supplemental search for individual RCTs that were published more recently (e.g., within the last 5 years, depending on the dates of the systematic reviews). By drawing on the work of previous systematic reviewers, this approach is less resource intensive, saving time and effort while still covering the available literature $(27,49)$. We will use the following databases to search from inception for reviews and meta-analyses: Cochrane Library, CINAHL, MEDLINE/PubMed, MEDLINE In-Process, EMBASE, and PsychINFO. We will extract RCTs and their associated data from the collected reviews and original studies. In addition, we will perform an updated search for relevant RCTs. We anticipate that the time-period for these searches will be within the last 3 - 5 years; however, the range will be determined by how recent the available systematic reviews are. We will use the databases MEDLINE/PubMed, the Cochrane Library and clinical trials registries (50) for RCT searches. The clinical trial registries we will include are the World Health Organization International Clinical Trials Registry Platform (WHO ICTRP) and clinicaltrials.gov. Furthermore, we will also search the reference lists of all included RCTs. Searches will adopt the BMJ trials and SR filters (available at: https://bestpractice.bmj.com/info/toolkit/learn-ebm/studydesign-search-filters/). Although searches will not be filtered by language, only English language articles will be included. We have provided a sample search strategy in Additional file 3. We will download references into the reference manager software Endnote, and remove duplicate references via the software tools. Two reviewers will independently select reviews and trials and review full-texts for inclusion, discussing disagreements with a third reviewer.

\section{Data extraction}

Data extraction will be completed using structured data extraction spreadsheets in Excel to obtain all relevant data in a consistent fashion. Double data entry will be carried out, whereby data will be inputted independently by two reviewers into two separate datasets and then compared. Details extracted from the data will include study characteristics (first author, year of publication, journal, setting, and country), participant characteristics (sample size, mean age, \% female, type of cancer, cancer stage [i.e., early-stage disease vs. advanced stage/palliative care], time since diagnosis, cancer treatment stage [i.e., pre-treatment, active treatment, post-treatment], depression inclusion criteria, baseline depression severity, depression assessment tools, presence of premorbid depression), and intervention and comparator group details (type of pharmacotherapy [name, dose, duration]; for psychotherapy interventions the Template for Intervention Description and Replication checklist [TIDiER] (51) will be used for extraction headings [i.e. intervention name, rationale/theory, materials, procedures, who delivered the intervention, delivery mode, location/setting, dose/intensity, tailoring, modifications, fidelity). Since psychotherapies can take many modalities and are delivered in different forms, the TIDiER headings will allow more precise documentation of any significant disparities among the selected psychotherapeutic treatments selected for study. Data extracted from the original RCT reports, including multi-arm trials, will be used to calculate summary effect sizes.

\section{Continuous outcomes}

For continuous outcomes, we will extract SMDs (when reported), 95\% confidence intervals, means, SDs and number of patients participating in trial arm of the study into the final dataset. If this information is not available, we will request these data from the RCT authors. If data are omitted in the reports (e.g., SDs not reported), we will impute using the Cochrane recommended techniques for estimating SDs (52) and the metaeff command procedure in Stata to calculate SMDs and 95\% confidence intervals from available data (53). If mean change scores are not reported, then we will consider outcome scores (27). If trials report the percentage of participants who no longer have elevated 
depression following the intervention, rather than mean change scores or outcome scores, we will also use the metaeff command to calculate SMD. If sufficient data are not available to calculate the SMD, we will include the study for descriptive purposes only and exclude it from the main NMA. If insufficient data are available to calculate the $95 \%$ confidence intervals, we will consider imputing based on the median from the other studies from that particular group $(27,29)$. We will carry out sensitivity analyses to determine whether there are implications of such imputations (27).

\section{Binary outcomes}

For the extraction of binary outcomes (i.e., acceptability, mortality, adverse events), we will obtain the number of participants with each event from each trial arm. When data are not available, we will contact the authors of the studies to request the information.

\section{Duration of RCTs and outcome assessments}

Following previous methods $(25,27,29,35)$ we will adopt an 8-week threshold for the synthesis of the primary depression outcome and the secondary QoL outcome. In those cases where data are unavailable for that duration, the closest available data from 4 to 12 weeks will be used (25). We will use overall dropout rate, regardless of timepoint as the second primary outcome for acceptability. We will use long-term depression assessments at 26 weeks (with an acceptable range between 20 and 30 weeks) as a secondary outcome.

\section{Missing RCT outcome data and units of analysis}

We will extract all data as they were reported in the original trials, regardless of how missing data were dealt with in each study. As part of our risk assessment, we will rate whether or not the handling of missing was appropriate or not, where possible drawing this information from previous systematic reviews $(27,54)$. In line with previous NMAs $(25$, $29,35)$, we will extract pertinent data to explain clustering from cluster randomised trials, and extract only data relating to the first study period from cross-over trials to prevent carry-over effects.

\section{Risk of bias and quality of evidence}

Two reviewers will independently extract and assess risk of bias (RoB) data, which will be used to inform updated RoB 2 tool (55) ratings. Of note, our use of the RoB 2 tool is likely to lead to fewer studies being classed as having a high RoB than existing systematic reviews that used the Cochrane tool (52), in particular for trials for which it was not possible to blind for treatment assignment. This is likely because the RoB 2 involves a more nuanced decisionmaking framework that does not automatically consider unblinded studies to be at high risk of bias (55). For example, for trials in which blinding was not feasible or implemented, the RoB 2 considers whether postrandomisation deviations from the intervention led to bias or whether the reasons for missing outcome data contributed to bias; if not, such trials can still meet the criteria for low RoB. If data to assess risk of bias are insufficient or missing, we will consider contacting RCT authors to obtain additional information. In instances when the two independent reviewers disagree on RoB ratings, input from a third reviewer will help to settle disagreements.

We will use the Confidence in Network Meta-Analysis (CINeMA) framework to evaluate the credibility of our results (56) and present the resulting information in a summary table. CINeMA, is based in part on the GRADE (Grading of Recommendations Assessment, Development and Evaluation) framework (57), but has been specifically developed to account for the complexity of NMA methods. The framework considers six domains: (i) within-study bias, (ii) reporting bias, (iii) indirectness, (iv) imprecision, (v) heterogeneity, and (vi) incoherence; and, as with GRADE, assessments of each domain are summarised to reflect whether confidence for the treatment effect is very low, low, 
moderate, or high (56). We will use CINeMA to summarise the strength of the evidence for each of the primary outcomes from the network estimates.

\section{Transitivity assessment}

Transitivity relates to how effect modifiers are distributed across intervention comparisons (23). To uphold this key assumption of NMA, studies making different direct comparisons must be sufficiently similar in all respects other than the intervention that is being examined (58), such that it is valid to make indirect comparisons between intervention groups that are connected via one or more intermediate comparator groups (34). Previous NMAs on the efficacy of antidepressants have identified that factors such as bipolarity, psychotic features and subthreshold depression can moderate the efficacy of antidepressants and therefore, to uphold the transitivity assumption of the network, have limited samples to non-psychotic patients with unipolar depression (35). We will take a similar approach and, in line with Doyle et al. (29), exclude studies where $20 \%$ or more of participants have bipolar or psychotic depression, or concurrent secondary psychiatric diagnoses. Another factor that may moderate the efficacy of interventions for depression is whether participants enrolled in an RCT have elevated depressive symptoms (i.e., score above a specified threshold on a validated measure of depression) at baseline. Including trails that do not specify elevated depression as an inclusion criteria may misrepresent the efficacy of interventions, because participants who have sub-threshold levels of depression to begin with have less scope to improve on this outcome (21). Indeed, previous studies have demonstrated that baseline severity of depression moderates the efficacy of psychosocial treatments for patients with cancer, such that effects are negligible when baseline depression is low (59). Therefore, we have specified that only RCTs that specifically enrolled participants with elevated depressive symptoms meet our inclusion criteria. Given these precautions, we assume that participants who fulfil the inclusion criteria for this protocol are equally eligible to be randomized to any of the intervention groups. Nevertheless, to assess transitivity and further guard against violating this assumption, we will compile a list of potential effect modifiers from data collected (e.g., participant age, sex, cancer type, treatment stage, time post-diagnosis, and cancer stage, level of depressive symptoms at baseline, the presence of other comorbidities), and investigate whether the distribution of these variables is similar across the studies included in pairwise comparisons (see Assessment of heterogeneity and inconsistency section for further details) (34).

\section{Statistical analysis}

We will use Stata 15 to carry out all quantitative analysis.

\section{Study and network characteristics}

We will present study characteristics and descriptive statistics on important clinical and methodological variables for all included RCTs (e.g., publication year, age, sex breakdown, settings, cancer type and severity, stage of treatment, etc.). We will generate network diagrams to illustrate the amount of evidence for each outcome and the properties of the RCTs contributing to each outcome (34). The size of each node in the diagrams will represent the number of participants in the intervention/comparator group, the edge width of the node will represent the number of RCTs involving a given intervention/comparator group, while lines connecting the nodes will signify the intervention/comparison groups that have been directly compared in the available RCTs (23).

\section{Pairwise meta-analysis}

We will perform random effects pairwise meta-analyses when head-to-head data are available. This will allow us to examine whether study characteristics are comparable across the RCTs that inform each direct comparison (see Heterogeneity and inconsistency assessments section), explore the impact of any potential outliers (60), and identify 
differences in estimated effects from the NMA that may be due to correlations between outcomes (29). For each pairwise analyses, we will report SMD or odds ratios, both with associated $95 \%$ confidence intervals, for continuous and binary outcomes respectively $(61,62)$.

\section{Heterogeneity and inconsistency assessments}

We will explore the impact of effect modifiers within and across comparisons, that may lead to heterogeneity and inconsistency respectfully, using both local and global measures (34). Specifically, we will use the design-bytreatment interaction model to assess inconsistency in networks as a whole (58). If we find evidence of inconsistency, we will contrast direct evidence with indirect evidence from specific loops (loop-specific) and from the entire network (node-specific) to detect pairwise comparisons or loops of evidence that may be introducing inconsistency in the network locally $(34,58)$. We will graphically present effect sizes using Forest plots to explore the possibility of statistical heterogeneity. Furthermore, we will quantify statistical heterogeneity and statistical inconsistency for each pairwise meta-analysis using $R$.

\section{Network meta-analysis}

We will carry out a frequentist random-effects multivariate network meta-analysis to synthesize all evidence for each outcome and obtain a comprehensive ranking of all intervention groups for the primary outcomes. To this end, we will use the commands network meta and mvmeta (which underpins the first command) in Stata 15 (61). These commands use a Newton-Raphson procedure to carry out a restricted maximum likelihood (REML) multivariate metaanalysis, which accounts for within- and between-study correlations. Our analysis will include all available interventions types and comparator groups, as described and grouped above (i.e., pharmacology interventions, psychotherapy interventions etc.). If sufficient data are available, we will perform a second analysis that separates the various groupings by subtype (e.g., type of psychotherapy). We will use Rankograms and surface under the cumulative ranking (SUCRA) curves to rank intervention groups and visually present the uncertainty in ranking probabilities (34). An intervention/intervention groups' SUCRA value corresponds to the ratio of the area under the cumulative ranking curve to the entire area in the plot. As such, it refers to the percentage of effectiveness/acceptability of an intervention/intervention group relative to a hypothetical intervention that would be rated the best without any uncertainty (23).

\section{Sensitivity analysis}

We will perform sensitivity analyses to examine the robustness of our findings with regard to the primary outcomes by carrying out subgroup analysis for the following, provided sufficient data are available:

1) studies with different levels of bias (i.e., low, of some concern, high);

2) studies of patients who meet the criteria for depression on a diagnostic interview and patients who score above the cut-off on a validated measure of depression;

3) studies of patient groups at different stages of treatment (i.e., patients who are pre-treatment, on active treatment, or post-treatment);

4) Studies of patients with advanced/incurable cancers or receiving palliative care (i.e., advanced cancer vs. earlystage disease);

5) Studies of patients with different cancer types (e.g., breast cancer vs. other cancers);

6) Studies that include patients with a premorbid history of depression and. those who do not.

\section{Bias assessments}


We will consider the likelihood that studies were conducted and not published and the comprehensiveness of our search strategy in evaluating the possibility of publication bias. We will use Funnel plots and Egger's test to evaluate publication bias and the influence of smaller studies in pair-wise comparisons $(34,58)$. Furthermore, we will assess asymmetry on comparison-adjusted funnel plots, to examine possible associations between study size and study effect (23). Comparison-adjusted funnel plots graph the inverted standard error of the effect size on one axis to an adjusted effect size, which comprises the difference between a study estimate and their direct meta-analysis mean effect, and can be used to examine whether results vary depending on trial precision in NMAs $(34,58)$. Finally, we will carry out a network-meta regression to determine associations between study size and effect size (63).

\section{Discussion}

The findings from this NMA will provide the best available evidence for managing depression among patients with cancer, and provide treatment rankings in terms of both efficacy and acceptability. In addition, our findings will give insight into whether diverse management approaches differentially impact on quality of life and mortality, as well as rates of adverse events associated with treatments. Such information will help to inform clinical guidelines and be of key importance to healthcare professionals and patients, helping them to make informed and evidence-based decisions about what treatment approach, or combination of approaches, is likely to benefit them most. This information will also be valuable for policy-makers, funders, and health service providers in informing the development of psycho-oncological services and service configuration. Furthermore, our review will help to identify existing gaps in the literature, by highlighting available interventions for managing depression among patients with cancer for which there is currently insufficient evidence because of a lack of available research.

\section{Abbreviations}

CINeMA

Confidence in NEtwork Meta-Analysis

CAM

Complementary and alternative medicine

DSM5

Diagnostic and Statistical Manual of Mental Disorders, 5th Edition

GRADE

Grading of Recommendations Assessment, Development and Evaluation framework

GRIPP2-SF

Guidance for Reporting Involvement of Patients and the Public 2 - Short Form

HAM-D

Hamilton Depression Rating Scale

HRQoL

Health-related quality of life

NMA

Network meta-analysis

PICO

Patient, Intervention, Comparison, Outcome

PPI

Public and Patient Involvement

RCTs 
Randomised controlled trials

REML

Restricted maximum likelihood

RoB

Risk of Bias

SDs

standard deviations

SMD

Standardised mean difference

SUCRA

Surface under the cumulative ranking

TIDiER

Template for intervention description and replication checklist

TAU

Treatment as usual

WHO ICTRP

World Health Organization International Clinical Trials Registry Platform

\section{Declarations}

\section{Ethics approval and consent to participate:}

Not applicable

\section{Consent for publication}

Not applicable

\section{Availability of data and materials}

Not applicable

\section{Competing interests}

The authors declare that they have no competing interests.

The funder did not play a role in in developing the protocol.

\section{Funding}

This work is supported by Irish Cancer Society and National Cancer Control Programme Award PSY19PER.

\section{Authors' contributions}

MP and FD conceived of the idea for the manuscript. MP and SP wrote the first draft. FD made substantial contributions to developing the protocol. All authors were involved in refining the draft manuscript or providing critical input to revise it. All authors provided final approval for submission. MP is the guarantor of the review.

\section{Acknowledgements}


The project is funded by a Psycho-Oncology Research Award, a collaborative grant between the Irish Cancer Society and the National Cancer Control Programme (NCCP), which aims to stimulate, develop, and support psycho-oncology research in Ireland. We thank the Irish Cancer Society PPI panel for their valuable review of the study proposal and their recommendations. We are grateful for the ongoing input of the ENHANCE PPI panel, Katie Verling and Garret Monahan, for their input into the study design and the running of the project. We thank Mr. Paul Murphy, information specialist in the RCSI Library, for his advice on and help in developing the search terms.

\section{References}

1. Hartung T, Brähler E, Faller H, Härter M, Hinz A, Johansen C, et al. The risk of being depressed is significantly higher in cancer patients than in the general population: Prevalence and severity of depressive symptoms across major cancer types. Eur J Can. 2017;72:46-53.

2. Walker J, Hansen CH, Martin P, Symeonides S, Ramessur R, Murray G, et al. Prevalence, associations, and adequacy of treatment of major depression in patients with cancer: a cross-sectional analysis of routinely collected clinical data. Lancet Psychiatry. 2014;1(5):343-50.

3. Mitchell AJ, Chan M, Bhatti H, Halton M, Grassi L, Johansen C, et al. Prevalence of depression, anxiety, and adjustment disorder in oncological, haematological, and palliative-care settings: a meta-analysis of 94 interviewbased studies. Lancet Oncology. 2011;12(2):160-74.

4. Miller K, Massie MJ. Depressive disorders. Psycho-Oncol. 2010;2:311-8.

5. Massie MJ. Prevalence of depression in patients with cancer. J Nat Can Inst. 2004(32):57-71.

6. Breitbart WS, Alici Y. Psycho-oncology. Harvard Rev of Psych. 2009;17(6):361-76.

7. Smith HR. Depression in cancer patients: Pathogenesis, implications and treatment. Oncol Lett. 2015;9(4):150914.

8. Niedzwiedz CL, Knifton L, Robb KA, Katikireddi SV, Smith DJ. Depression and anxiety among people living with and beyond cancer: a growing clinical and research priority. BMC Cancer. 2019;19(1):1-8.

9. Wang Y-H, Li J-Q, Shi J-F, Que J-Y, Liu J-J, Lappin JM, et al. Depression and anxiety in relation to cancer incidence and mortality: a systematic review and meta-analysis of cohort studies. Mol Psych. 2020;25(7):1487-99.

10. Mols F, Husson O, Roukema J-A, van de Poll-Franse LV. Depressive symptoms are a risk factor for all-cause mortality: results from a prospective population-based study among 3,080 cancer survivors from the PROFILES registry. J Can Surviv. 2013;7(3):484-92.

11. Pinquart M, Duberstein PR. Depression and cancer mortality: A meta-analysis. Psychol Med. 2010;40(11):1797810.

12. Arrieta O, Angulo LP, Núñez-Valencia C, Dorantes-Gallareta Y, Macedo EO, Martínez-López D, et al. Association of depression and anxiety on quality of life, treatment adherence, and prognosis in patients with advanced nonsmall cell lung cancer. Ann Surg Oncol. 2013;20(6):1941-8.

13. Laoutidis ZG, Mathiak K. Antidepressants in the treatment of depression/depressive symptoms in cancer patients: A systematic review and meta-analysis. BMC Psychiatry. 2013;13.

14. Li M, Fitzgerald P, Rodin G. Evidence-based treatment of depression in patients with cancer. J Clin Oncol. 2012;30(11):1187-96.

15. Feng Y, Wang X-Y, Li S-D, Zhang Y, Wang H-M, Min L, et al. Clinical research of acupuncture on malignant tumor patients for improving depression and sleep quality. J Trad Chinese Med. 2011;31(3):199-202.

Page $14 / 18$ 
16. Walker J, Sawhney A, Hansen $\mathrm{CH}$, Ahmed S, Martin P, Symeonides S, et al. Treatment of depression in adults with cancer: a systematic review of randomized controlled trials. Psychol Med. 2014;44(5):897-907.

17. Craft LL, Vaniterson EH, Helenowski IB, Rademaker AW, Courneya KS. Exercise effects on depressive symptoms in cancer survivors: a systematic review and meta-analysis. Cancer Epidemiol Biomarkers Prev. 2012;21(1):3-19.

18. Ostuzzi G, Matcham F, Dauchy S, Barbui C, Hotopf M. Antidepressants for the treatment of depression in people with cancer. Cochrane Database Syst Rev. 2018;4:CD011006.

19. Li M, Kennedy EB, Byrne N, Gérin-Lajoie C, Katz MR, Keshavarz H, et al. Management of depression in patients with cancer: a clinical practice guideline. Journal of Oncol Pract. 2016;12(8):747-56.

20. Roy-Byrne PP. Management of psychiatric disorders in patients with cancer. 2021. In: UpToDate [Internet]. Available from: https://www.uptodate.com/contents/management-of-psychiatric-disorders-in-patients-withcancer?

search=depression\%20cancer\&source=search_result\&selectedTitle=1 150\&usage_type=default\&display_rank=1.

21. Hart SL, Hoyt MA, Diefenbach M, Anderson DR, Kilbourn KM, Craft LL, et al. Meta-analysis of efficacy of interventions for elevated depressive symptoms in adults diagnosed with cancer. J Nat Can Inst. 2012;104(13):990-1004.

22. Osborn RL, Demoncada AC, Feuerstein M. Psychosocial interventions for depression, anxiety, and quality of life in cancer survivors: meta-analyses. Int J Psych Med. 2006;36(1):13-34.

23. Mavridis D, Giannatsi M, Cipriani A, Salanti G. A primer on network meta-analysis with emphasis on mental health. Evid Based Ment Health. 2015;18(2):40-6.

24. Leucht S, Chaimani A, Cipriani AS, Davis JM, Furukawa TA, Salanti G. Network meta-analyses should be the highest level of evidence in treatment guidelines. Eur Arch Psychiatry Clin Neurosci. 2016;266(6):477-80.

25. Cipriani A, Furukawa TA, Salanti G, Chaimani A, Atkinson LZ, Ogawa Y, et al. Comparative efficacy and acceptability of 21 antidepressant drugs for the acute treatment of adults with major depressive disorder: A systematic review and network meta-analysis. Lancet. 2018;391(10128):1357-66.

26. Noone C, Leahy J, Morrissey EC, Newell J, Newell M, Dwyer CP, et al. Comparative efficacy of exercise and antihypertensive pharmacological interventions in reducing blood pressure in people with hypertension: a network meta-analysis. European J of Prev Card. 2020;27(3):247-55.

27. Doyle F, Freedland KE, Carney RM, de Jonge P, Dickens C, Pedersen SS, et al. Hybrid systematic review and network meta-analysis of randomized controlled trials of interventions for depressive symptoms in patients with coronary artery disease. Psychosom Med. 2021;83(5):423-31.

28. American Psychiatric Association. Diagnostic and statistical manual of mental disorders. 5th ed. Washington, D.C.: American Psychiatric Publishing; 2013.

29. Doyle F, Freedland K, Carney R, de Jonge P, Dickens C, Pedersen S, et al. Network meta-analysis of randomised trials of pharmacological, psychotherapeutic, exercise and collaborative care interventions for depressive symptoms in patients with coronary artery disease: hybrid systematic review of systematic reviews protocol. Syst Rev. 2019;8(1):71.

30. Hutton B, Salanti G, Caldwell DM, Chaimani A, Schmid CH, Cameron C, et al. The PRISMA extension statement for reporting of systematic reviews incorporating network meta-analyses of health care interventions: checklist and explanations. Ann Intern Med. 2015;162(11):777-84.

31. Staniszewska S, Brett J, Simera I, Seers K, Mockford C, Goodlad S, et al. GRIPP2 reporting checklists: tools to improve reporting of patient and public involvement in research. Res Involv Engagem. 2017;3(1):13.

Page 15/18 
32. National Institute for Health and Clinical Excellence. Depression in adults with a chronic physical health problem. NICE clinical guideline 91 (CG91). 2009. Available from: www.nice.org.uk/nicemedia/pdf/CG91FullGuideline.pdf.

33. Campbell KL, Winters-Stone KM, Wiskemann J, May AM, Schwartz AL, Courneya KS, et al. Exercise guidelines for cancer survivors: consensus statement from international multidisciplinary roundtable. Med Sci Sports Exerc. 2019;51(11):2375-90.

34. Chaimani A, Caldwell DM, Li T, Higgins JP, Salanti G. Additional considerations are required when preparing a protocol for a systematic review with multiple interventions. J Clin Epidemiol. 2017;83:65-74.

35. Furukawa TA, Salanti G, Atkinson LZ, Leucht S, Ruhe HG, Turner EH, et al. Comparative efficacy and acceptability of first-generation and second-generation antidepressants in the acute treatment of major depression: protocol for a network meta-analysis. BMJ Open. 2016;6(7):e010919.

36. Cuijpers P, Reijnders M, Karyotaki E. Psychological treatment of depression: An updated meta-analytic database of randomized trials. Submitted for publication [Internet]. 2018. Available from: https://evidencebasedpsychotherapies.shinyapps.io/metapsy/_w_ed60cf71/protocol.pdf.

37. Campbell LF, Norcross JC, Vasquez MJT, Kaslow NJ. Recognition of psychotherapy effectiveness: The APA resolution. Psychother. 2013;50(1):98-101.

38. Sasso JP, Eves ND, Christensen JF, Koelwyn GJ, Scott J, Jones LW. A framework for prescription in exerciseoncology research. J Cachexia Sarcopenia Muscle. 2015;6(2):115-24.

39. Bland KA, Neil-Sztramko SE, Zadravec K, Medysky ME, Kong J, Winters-Stone KM, et al. Attention to principles of exercise training: an updated systematic review of randomized controlled trials in cancers other than breast and prostate. BMC Cancer. 2021;21(1):1179.

40. Li M, Kennedy EB, Byrne N, Gérin-Lajoie C, Katz MR, Keshavarz H, et al. Systematic review and meta-analysis of collaborative care interventions for depression in patients with cancer. Psycho-Oncol. 2017;26(5):573-87.

41. National Center for Complementary and Integrative Health. Complementary, Alternative, or Integrative Health: What's In a Name? 2016 [Available from: https://nccih.nih.gov/health/integrative-health.

42. Haller H, Anheyer D, Cramer H, Dobos G. Complementary therapies for clinical depression: an overview of systematic reviews. BMJ Open. 2019;9(8):e028527-e.

43. van der Watt G, Laugharne J, Janca A. Complementary and alternative medicine in the treatment of anxiety and depression. Curr Opin Psychiatry. 2008;21(1):37-42.

44. Levack WM, Martin RA, Graham FP, Hay-Smith. Compared to what? An analysis of the management of control groups in Cochrane reviews in neurorehabilitation. Eur J Phys Rehabil Med. 2019;55(3):353-63.

45. Karlsson P, Bergmark A. Compared with what? An analysis of control-group types in Cochrane and Campbell reviews of psychosocial treatment efficacy with substance use disorders. Addiction. 2015;110(3):420-8.

46. Gold SM, Enck P, Hasselmann H, Friede T, Hegerl U, Mohr DC, et al. Control conditions for randomised trials of behavioural interventions in psychiatry: a decision framework. Lancet Psychiatry. 2017;4(9):725-32.

47. Doyle F, Conroy R, McGee H. Challenges in reducing depression-related mortality in cardiac populations: cognition, emotion, fatigue or personality? Health Psychol Rev. 2007;1(2):137-72.

48. Williams S, Dale J. The effectiveness of treatment for depression/depressive symptoms in adults with cancer: a systematic review. Br J Cancer. 2006;94(3):372-90.

49. Doyle F, Dempster M, editors. Hybrid systematic review for network meta-analysis: the good, the bad and the ugly. European Health Psychology Society Conference; 2021; Online. 
50. Pollock M, Fernandes RM, Becker LA, Featherstone R, Hartling L. What guidance is available for researchers conducting overviews of reviews of healthcare interventions? A scoping review and qualitative metasummary. Syst Rev. 2016;5(1):190.

51. Hoffmann TC, Glasziou PP, Boutron I, Milne R, Perera R, Moher D, et al. Better reporting of interventions: template for intervention description and replication (TIDieR) checklist and guide. BMJ. 2014;348:g1687.

52. Higgins J, Green S, Altman D, Sterne J. Cochrane handbook for systematic reviews of interventions version 510. 2011. The Cochrane Collaboration. Available from: http://handbook-5-1.cochrane.org/.

53. Kontopantelis E, Reeves D. METAEFF: Stata module to perform effect sizes calculations for meta-analyses. Statistical Software Components S457072: Boston College Department of Economics; 2009.

54. Tully PJ, Baumeister H. Collaborative care for comorbid depression and coronary heart disease: a systematic review and meta-analysis of randomised controlled trials. BMJ Open. 2015;5(12):e009128.

55. Sterne JAC, Savović J, Page MJ, Elbers RG, Blencowe NS, Boutron I, et al. RoB 2: a revised tool for assessing risk of bias in randomised trials. BMJ. 2019;366(366):I4898.

56. Nikolakopoulou A, Higgins JPT, Papakonstantinou T, Chaimani A, Del Giovane C, Egger M, et al. CINeMA: An approach for assessing confidence in the results of a network meta-analysis. PLOS Medicine. 2020;17(4):e1003082.

57. Guyatt GH, Oxman AD, Vist GE, Kunz R, Falck-Ytter Y, Alonso-Coello P, et al. GRADE: an emerging consensus on rating quality of evidence and strength of recommendations. BMJ. 2008;336(7650):924-6.

58. Salanti G, Del Giovane C, Chaimani A, Caldwell DM, Higgins JP. Evaluating the quality of evidence from a network meta-analysis. PLoS One. 2014;9(7):e99682.

59. Schneider S, Moyer A, Knapp-Oliver S, Sohl S, Cannella D, Targhetta V. Pre-intervention distress moderates the efficacy of psychosocial treatment for cancer patients: a meta-analysis. J Behav Med. 2010;33(1):1-14.

60. Jackson D, Riley R, White IR. Multivariate meta-analysis: potential and promise. Stat Med. 2011;30(20):2481-98.

61. White I. NETWORK: Stata module to perform network meta-analysis. Statistical software components S458319: Boston College Department Department of Economics; 2018.

62. Tak LM, Meijer A, Manoharan A, de Jonge P, Rosmalen JG. More than the sum of its parts: meta-analysis and its potential to discover sources of heterogeneity in psychosomatic medicine. Psychosom Med. 2010;72(3):253-65.

63. Mavridis D, Welton NJ, Sutton A, Salanti G. A selection model for accounting for publication bias in a full network meta-analysis. Stat Med. 2014;33(30):5399-412.

\section{Figures}




\section{Possible network plot}

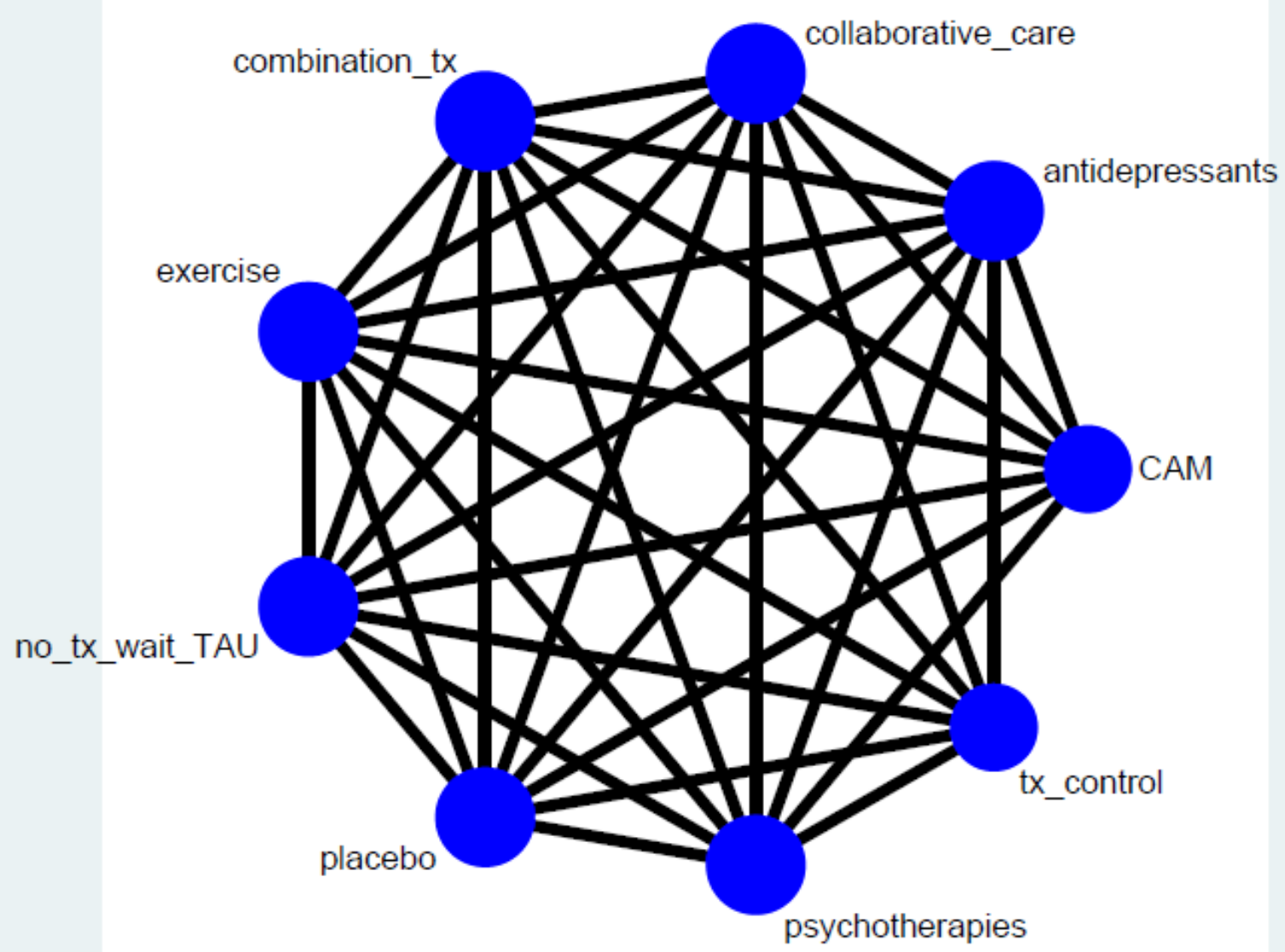

Figure 1

Sample network of all possible depression intervention and control group comparisons.

\section{Supplementary Files}

This is a list of supplementary files associated with this preprint. Click to download.

- PertIENHANCEAdditionalfile1PRISMAP.docx

- PertlENHANCEAdditionalfile2GRIPP2SF.docx

- PertIENHANCEAdditionalfile3SearchStrategy.docx 